\title{
THERMOELASTIC RESPONSE OF NANOBEAM RESONATORS SUBJECTED TO EXPONENTIAL DECAYING TIME VARYING LOAD
}

\author{
Ahmed E. Abouelregal \\ Mansoura University, Faculty of Science, Department of Mathematics, Mansoura, Egypt \\ e-mail: ahabogal@gmail.com \\ Ashraf M. Zenkour \\ King Abdulaziz University, Faculty of Science, Department of Mathematics, Jeddah, Saudi Arabia and \\ Kafrelsheikh University, Faculty of Science, Department of Mathematics, Kafrelsheikh, Egypt \\ e-mail:zenkour@kau.edu.sa; zenkour@sci.kfs.edu.eg
}

\begin{abstract}
This work investigates the vibrational response of thermoelastic nanobeam resonators induced by ramp-type heating and subjected to exponential decaying time varying load via Euler-Bernoulli beam theory. Governing equations are derived in the context of nonlocal generalized thermoelasticity theory with dual phase lags. The nonlocal nanobeam theory incorporates a nonlocal parameter to capture the small scale effect. Using the Laplace transform technique, an analytical solution has been attained. and inversions of the transformed solutions have been carried out by means of calculus of residues. The effects of nonlocal, point load and ramping-time parameters on all studied fields of the nanobeam are investigated and discussed.
\end{abstract}

Keywords: thermoelasticity, nonlocal nanobeam, varying load, ramp-type heating

\section{Introduction}

The fields of micro-, electro-, and mechanical systems (MEMS) have become quickly and gone into many resistances and correspondence technologies. Advanced applications for fabricating a variety of MEMS gadgets have been created to deal with all requirements for industries. It is known that all MEMS systems have mechanical flexible components. Microscales of cantilevers, bridges and membranes with various geometrical measurements and arrangements that often carry load are considered as MEMS systems (Younis, 2011). For MEMS designers, it is essential to understand mechanical properties of flexible micro-devices keeping in mind the end goal to predict the amount of transverse displacement from a distributed load and the other way around to forestall cracking-fracture, improve performance and to increase lifetime of MEMS gadgets (Allameh, 2003).

Both investigational and atomistic reproduction computations have demonstrated a significant dimension influence in mechanical properties when the sizes of such structures become very small. For this purpose, the size influence has a crucial role in dynamic and static behavior of micro-/nano-structures and cannot be neglected. It is famous that classical continuum mechanics does not represent such dimension influences in microscale and nanoscale structures. It is well known in the classical (local) elasticity theory that the stress at a point depends just on the strain at the same point. That is not the same in nonlocal elasticity theory, in which the stress at a point may be a function of strains at all points in the solid.

The nonlocal elasticity theory of Eringen $(1983,2002)$ has been increasingly used to deal with nanostructures as a reliable and quick technique. Nonlocal elasticity has been applied to micro- and nanomaterials and it recently received much attention among nanotechnology. The 
basic difference between both classical and nonlocal elasticity theories is based on the definition of stress. Eringen's nonlocal theory includes more information about long range forces about atoms and, thus, internal scale length is introduced (Arefi and Zenkour, 2016; Zenkour, 2016a,c; Zenkour and Abouelregal, 2014a,b, 2015, 2016).

Lord and Shulman (LS) (1967) presented generalized theory of thermoelasticity with first relaxation time for isotropic homogeneous materials, in which an altered law of heat conduction that incorporates both time derivative of the heat flux and the heat flux itself, replaces Fourier's law conventional. The heat equation associated with LS theory is of hyperbolic type and consequently eliminates the paradox of infinite velocity of propagation inherent in both coupled (CTE) and uncoupled theories of thermoelasticity. Tzou $(1995,1997)$ presented another alteration with Fourier's law to investigate two time lags (see also Abbas and Zenkour, 2014; Abouelregal, 2011; Abouelregal and Abo-Dahab, 2012; Zenkur, 2016b; Zenkour et al., 2013).

The objective of this paper is to derive governing equations of motion for free vibration of nonlocal Euler-Bernoulli nanobeams subjected to time-varying transverse load. In this work, a thermoelastic model based on the dual-phase-lag modification (DPL) heat conduction equation is used. The Laplace transform method and its inversion is used in the derivation. The effects due to nonlocal, point load and ramping time parameters will be studied. The current model may be used in micro-electro-mechanical applications such as frequency filters, relay switches, accelerometers, mass flow sensors and resonators.

\section{Mathematical model and problem formulation}

A schematic diagram of a thin elastic nanobeam is illustrated in Fig. 1 in which geometrical parameters of length $(0 \leqslant x \leqslant L)$, width $b(-b / 2 \leqslant y \leqslant b / 2)$ and uniform thickness $h$ $(-h / 2 \leqslant z \leqslant h / 2)$ are also indicated. We take $x$-axis along the axis of the nanobeam and $y$ and $z$-axes correspond to width and thickness, respectively. The nanobeam is considered to be unstrained, unstressed and at environment temperature $T_{0}$ in equilibrium.

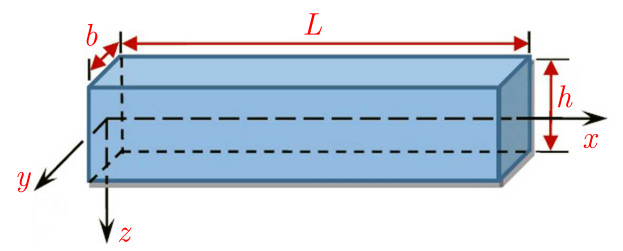

Fig. 1. Schematic diagram of the nanobeam

The linear Euler-Bernoulli theory is used to investigate bending vibration of the nanobeam. Any plane cross-section in the beginning perpendicular to the axis of the nanobeam remains plane and perpendicular to the neutral surface through bending. Hence, displacements of any point of the nanobeam can be written as

$$
u=-z \frac{\partial w}{\partial x} \quad v=0 \quad w=w(x, t)
$$

in which $w$ represents the lateral transverse deflection.

According to Eringen's nonlocal theory of elasticity (Eringen, 1983), with the aid of Eq. (2.1), the one-dimensional constitutive relation can be simplified to

$$
\sigma_{x}-\xi \frac{\partial^{2} \sigma_{x}}{\partial x^{2}}=-E\left(z \frac{\partial^{2} w}{\partial x^{2}}+\alpha_{T} \theta\right)
$$

where $\sigma_{x}$ is the nonlocal axial stress, $\alpha_{T}=\alpha_{t} /(1-2 \nu)$ in which $\alpha_{t}$ is the thermal expansion coefficient and $\nu$ is Poisson's ratio, $\xi=\left(e_{0} a\right)^{2}$ represents the nonlocal parameter in which 
$a$ is internal characteristic length and $e_{0}$ is a constant appropriate to each material and being determined by experiment. It can be observed that when the parameter $a$ is ignored, i.e., the elements of a medium are considered to be continuously distributed, then $\xi=0$, and then Eq. (2.2) may be reduced to be the constitutive equation of the classical case. Then, the bending moment of cross-section may be represented as

$$
M(x, t)=\int_{-h / 2}^{h / 2} z \sigma_{x} d z
$$

Upon using Eqs. (2.2) and (2.3), we obtain

$$
M(x, t)-\xi \frac{\partial^{2} M}{\partial x^{2}}=-E I\left(\frac{\partial^{2} w}{\partial x^{2}}+\alpha_{T} M_{T}\right)
$$

in which $I=b h^{3} / 12$ represents inertia moment of the nanobeam cross-section, $E I$ represents flexural rigidity and $M_{T}$ is the moment of the beam due to presence of thermal effects, which is given by

$$
M_{T}=\frac{12}{h^{3}} \int_{-h / 2}^{h / 2} \theta(x, z, t) z d z
$$

If the nanobeam is subjected to a distributed transverse load $q(x, t)$, the equation of transverse motion will be in the following form (Zhang et al., 2005)

$$
\frac{\partial^{2} M}{\partial x^{2}}=-q(x, t)+\rho A \frac{\partial^{2} w}{\partial t^{2}}
$$

in which $A=b h$ represents the area of nanobeam cross section. The flexure moment can be determined from Eqs. (2.4) and (2.6) as

$$
M(x, t)=\xi\left(\rho A \frac{\partial^{2} w}{\partial t^{2}}-q\right)-E I\left(\frac{\partial^{2} w}{\partial x^{2}}+\alpha_{T} M_{T}\right)
$$

Eliminating the moment $M$ from Eq. (2.6) with the aid of Eq. (2.7), we get the equation of motion of the nanobeam as

$$
\left[\frac{\partial^{4}}{\partial x^{4}}+\frac{\rho A}{E I} \frac{\partial^{2}}{\partial t^{2}}\left(1-\xi \frac{\partial^{2}}{\partial x^{2}}\right)\right] w-\frac{1}{E I}\left(1-\xi \frac{\partial^{2}}{\partial x^{2}}\right) q+\alpha_{T} \frac{\partial^{2} M_{T}}{\partial x^{2}}=0
$$

The generalized heat conduction equation in terms of the constitutive relations in the context of Tzou theory (Tzou, 1995, 1997) of generalized (non-Fourier) thermoelasticity is given by

$$
\left(1+\tau_{\theta} \frac{\partial}{\partial t}\right)\left(K \theta_{, i}\right)_{, i}+\left(1+\tau_{q} \frac{\partial}{\partial t}\right)(\rho Q)=\left(1+\tau_{q} \frac{\partial}{\partial t}\right) \frac{\partial}{\partial t}\left(\rho C_{E} \theta+\gamma T_{0} e\right)
$$

where $K$ denotes the thermal conductivity, $C_{E}$ represents specific heat per unit mass at uniform strain, $Q$ is heat source, $\theta=T-T_{0}$ is the excess temperature distribution, in which $T_{0}$ denotes environmental temperature, $\tau_{q}$ denotes the phase-lag of heat flux, and $\tau_{\theta}$ denotes the phase-lag of gradient of temperature, and $e=\frac{\partial u}{\partial x}+\frac{\partial w}{\partial z}$ is volumetric strain. Substituting Eq. (2.1) into heat equation Eq. (2.9), without considering heat source $(Q=0)$, gives

$$
K\left(1+\tau_{\theta} \frac{\partial}{\partial t}\right)\left(\frac{\partial^{2}}{\partial x^{2}}+\frac{\partial^{2}}{\partial z^{2}}\right) \theta=\left(1+\tau_{q} \frac{\partial}{\partial t}\right) \frac{\partial}{\partial t}\left(\rho C_{E} \theta-\gamma T_{0} z \frac{\partial^{2} w}{\partial x^{2}}\right)
$$

Equations (2.8) and (2.10) describe the nonlocal thermoelasticity theory with phase lags. The classical thermoelasticity theory may be recovered by putting $\xi=0$ in the above equations. For $\tau_{\theta}=0$ and $\tau_{q}>0$, one obtains the Lord and Shulman model (LS), and the classical coupled theory (CTE) is also obtained when $\tau_{q}=\tau_{\theta}=0$. 


\section{General solution along the direction of thickness}

Let the temperature increment vary sinusoidally through-the-thickness of the nanobeam as

$$
\theta(x, z, t)=\Theta(x, t) \sin \left(\frac{\pi}{h} z\right)
$$

Using Eq. (3.1) in governing equations (2.7), (2.8) and (2.10), we obtain

$$
\begin{aligned}
& M(x, t)=\xi\left(\rho A \frac{\partial^{2} w}{\partial t^{2}}-q\right)-E I\left(\frac{\partial^{2} w}{\partial x^{2}}+\frac{24 T_{0} \alpha_{T}}{\pi^{2} h} \Theta\right) \\
& {\left[\frac{\partial^{4}}{\partial x^{4}}+\frac{\rho A}{E I} \frac{\partial^{2}}{\partial t^{2}}\left(1-\xi \frac{\partial^{2}}{\partial x^{2}}\right)\right] w-\frac{1}{E I}\left(1-\xi \frac{\partial^{2}}{\partial x^{2}}\right) q+\frac{24 \alpha_{T}}{\pi^{2} h} \frac{\partial^{2} \Theta}{\partial x^{2}}=0} \\
& \left(1+\tau_{\theta} \frac{\partial}{\partial t}\right)\left(\frac{\partial^{2} \Theta}{\partial x^{2}}-\frac{\pi^{2}}{h^{2}} \Theta\right)=\left(1+\tau_{q} \frac{\partial}{\partial t}\right) \frac{\partial}{\partial t}\left(\frac{\rho C_{E}}{K} \Theta-\frac{\gamma T_{0} \pi^{2} h}{24 K} \frac{\partial^{2} w}{\partial x^{2}}\right)
\end{aligned}
$$

For convenience, one can present the following dimensionless variables

$$
\left\{u^{\prime}, w^{\prime}, x^{\prime}, z^{\prime}, L^{\prime}, b^{\prime}, h^{\prime}\right\}=\eta c\{u, w, x, z, L, b, h\} \quad\left\{t^{\prime}, \tau_{q}^{\prime}, \tau_{\theta}^{\prime}\right\}=\eta c^{2}\left\{t, \tau_{q}, \tau_{\theta}\right\}
$$

and

$$
\begin{array}{lll}
\Theta^{\prime}=\frac{1}{T_{0}} \Theta & \xi^{\prime}=\eta^{2} c^{2} \xi & M=\frac{1}{\eta c E I} M \\
q^{\prime}=\frac{A}{E I} q & c^{2}=\frac{E}{\rho} & \eta=\frac{\rho C_{E}}{K}
\end{array}
$$

Upon introducing the above dimensionless quantities into the governing equations, we can obtain (dropping the primes for convenience)

$$
\begin{aligned}
& M(x, t)=\frac{12 \xi}{h^{2}} \frac{\partial^{2} w}{\partial t^{2}}-\xi q-\frac{\partial^{2} w}{\partial x^{2}}-\frac{24 T_{0} \alpha_{T}}{\pi^{2} h} \Theta \\
& {\left[\frac{\partial^{4}}{\partial x^{4}}+\frac{12}{h^{2}} \frac{\partial^{2}}{\partial t^{2}}\left(1-\xi \frac{\partial^{2}}{\partial x^{2}}\right)\right] w-\left(1-\xi \frac{\partial^{2}}{\partial x^{2}}\right) q+\frac{24 T_{0} \alpha_{T}}{\pi^{2} h} \frac{\partial^{2} \Theta}{\partial x^{2}}=0} \\
& \left(1+\tau_{\theta} \frac{\partial}{\partial t}\right)\left(\frac{\partial^{2} \Theta}{\partial x^{2}}-\frac{\pi^{2}}{h^{2}} \Theta\right)=\left(1+\tau_{q} \frac{\partial}{\partial t}\right) \frac{\partial}{\partial t}\left(\Theta-\frac{\gamma \pi^{2} h}{(24 K \eta} \frac{\partial^{2} w}{\partial x^{2}}\right)
\end{aligned}
$$

Now, we consider an exponentially decaying time varying load in the form

$$
q(x, t)=-q_{0}\left(1-\delta \mathrm{e}^{-\Omega t}\right)
$$

where $q_{0}$ is the dimensionless magnitude of the point load and $\Omega$ represents the dimensionless frequency of the applied load, respectively ( $\delta=0$ for the uniformly distributed load).

Initially, the considered nanobeam has been assumed to be homogeneous, at rest, undeformed and at uniform temperature $T_{0}$. So, the dimensionless initial conditions of the problem may be reconsidered as

$$
\left.w(x, t)\right|_{t=0}=\left.\frac{\partial w(x, t)}{\partial t}\right|_{t=0}=\left.0 \quad \Theta(x, t)\right|_{t=0}=\left.\frac{\partial \Theta(x, t)}{\partial t}\right|_{t=0}=0
$$

The above conditions can be completed by adding other conditions at the ends of the nanobeam. Let these ends satisfy the following simply-supported boundary conditions

$$
\left.w(x, t)\right|_{x=0, L}=\left.0 \quad \frac{\partial^{2} w(x, t)}{\partial x^{2}}\right|_{x=0, L}=0
$$


Let us also consider that the nanobeam is thermally loaded on the boundary $x=0$. Then, according to Eq. (3.1), we can put

$$
\Theta=\theta_{0} f(x, t) \quad \text { on } \quad x=0
$$

in which $\theta_{0}$ is a constant and $f(x, t)$ is a varying ramp-type function with time described mathematically as follows

$$
\left.f(x, t)\right|_{x=0}= \begin{cases}0 & \text { for } \quad t \leqslant 0 \\ \frac{t}{t_{0}} & \text { for } \quad 0 \leqslant t \leqslant t_{0} \\ 1 & \text { for } \quad t>t_{0}\end{cases}
$$

in which $t_{0}$ represents a positive constant called the ramp-type parameter of the same dimensionless as time $t$. Also, the temperature at the edge of the nanobeam satisfies the relation

$$
\left.\frac{\partial \Theta}{\partial x}\right|_{x=L}=0
$$

\section{Laplace transform space solution}

If we apply the Laplace transform method to both sides of Eqs. (3.5), we can get

$$
\begin{aligned}
& \bar{M}(x, s)=-\left(\frac{d^{2}}{d x^{2}}-A_{3} s^{2}\right) \bar{w}-A_{2} \bar{\Theta}+\xi \bar{g}(s) \\
& {\left[\frac{d^{4}}{d x^{4}}-A_{3} s^{2} \frac{d^{2}}{d x^{2}}+A_{1} s^{2}\right] \bar{w}=-A_{2} \frac{d^{2} \bar{\Theta}}{d x^{2}}-\bar{g}(s)} \\
& \left(\frac{d^{2}}{d x^{2}}-B_{1}\right) \bar{\Theta}=-B_{2} \frac{d^{2} \bar{w}}{d x^{2}}
\end{aligned}
$$

where

$$
\begin{array}{lll}
A_{1}=\frac{12}{h^{2}} \quad A_{2}=\frac{24 T_{0} \alpha_{T}}{\pi^{h}} \quad A_{3}=\xi A_{1} & A_{4}=\frac{\pi^{2}}{h^{2}} \quad A_{5}=\frac{\gamma \pi^{2} h}{24 K \eta} \\
B_{1}=A_{4}+\frac{s\left(1+\tau_{q} s\right)}{1+\tau_{\theta} s} & B_{2}=\frac{s\left(1+\tau_{q} s\right)}{1+\tau_{\theta} s} A_{5} & \bar{g}(s)=q_{0}\left(\frac{1}{s}-\frac{\delta}{\Omega+s}\right)
\end{array}
$$

Eliminating the function $\bar{\Theta}$ from Eqs. $(4.1)_{2}$ and $(4.1)_{3}$, we get the six-order differential equation for $\bar{w}$ in the form

$$
\left[\frac{d^{6}}{d x^{6}}-A \frac{d^{4}}{d x^{4}}+B \frac{d^{2}}{d x^{2}}-C\right] \bar{w}=0
$$

where

$$
A=B_{1}+A_{2} B_{2}+A_{3} s^{2} \quad B=s^{2}\left(A_{1}+A_{3} B_{1}\right) \quad C=A_{1} B_{1} s^{2}
$$

The general solution of $\bar{w}$ can be obtained as

$$
\bar{w}(x, s)=\sum_{j=1}^{3}\left(C_{j} \mathrm{e}^{-m_{j} x}+C_{j+3} \mathrm{e}^{m_{j} x}\right)
$$

where $m_{1}^{2}, m_{2}^{2}$ and $m_{3}^{2}$ represent roots of the characteristic equation

$$
m^{6}-A m^{4}+B m^{2}-C=0
$$


Substituting Eq. (4.1) $)_{3}$ into Eq. (4.1) $)_{2}$, leads to

$$
\bar{\Theta}(x, s)=-\frac{1}{A_{2} B_{1}}\left[\frac{d^{4} \bar{w}}{d x^{4}}-\left(A_{2} B_{2}+A_{3} s^{2}\right) \frac{d^{2} \bar{w}}{d x^{2}}+A_{1} s^{2} \bar{w}+\bar{g}(s)\right]
$$

The general solution to Eq. (4.7) with the help of Eq. (4.5) can be simplified as

$$
\bar{\Theta}(x, s)=\sum_{j=1}^{3} H_{j}\left(C_{j} \mathrm{e}^{-m_{j} x}+C_{j+3} \mathrm{e}^{m_{j} x}\right)-H_{4}
$$

where

$$
H_{j}=-\frac{1}{A_{2} B_{1}}\left[m_{j}^{4}-\left(A_{2} B_{2}+A_{3} s^{2}\right) m_{j}^{2}+A_{1} s^{2}\right] \quad H_{4}=\frac{\bar{g}(s)}{A_{2} B_{1}}
$$

Substituting the expressions of $\bar{w}$ and $\bar{\Theta}$ from Eqs. (4.5) and (4.8) into Eq. (4.1) 1 , we get the solution for the bending moment $\bar{M}$ as

$$
\bar{M}(x, s)=-\sum_{j=1}^{3}\left(m_{j}^{2}-A_{3} s^{2}+A_{2} H_{j}\right)\left(C_{j} \mathrm{e}^{-m_{j} x}+C_{j+3} \mathrm{e}^{m_{j} x}\right)+A_{2} H_{4}+\xi \bar{g}(s)
$$

In addition, the axial displacement $\bar{u}$ after using the deflection $\bar{w}(x, s)$ appearing in Eq. (4.5) can be expressed as

$$
\bar{u}=-z \frac{d \bar{w}}{d x}=z \sum_{j=1}^{3} m_{j}\left(C_{j} \mathrm{e}^{-m_{j} x}-C_{j+3} \mathrm{e}^{m_{j} x}\right)
$$

Finally, the boundary conditions in Eqs. (3.8), (3.9) and (3.11) in the Laplace transform domain are reduced to

$$
\begin{aligned}
& \left.\bar{w}(x, s)\right|_{x=0, L}=\left.0 \quad \frac{d^{2} \bar{w}(x, s)}{d x^{2}}\right|_{x=0, L}=0 \\
& \left.\bar{\Theta}(x, s)\right|_{x=0}=\theta_{0}\left(\frac{1-\mathrm{e}^{-t_{0} s}}{t_{0} s^{2}}\right)=\left.\bar{G}(s) \quad \frac{d \bar{\Theta}}{d x}\right|_{x=L}=0
\end{aligned}
$$

The above boundary conditions are applied to Eqs. (4.5) and (4.8) to determine the unknown parameters $C_{j}$ and $C_{j+3}$ as

$$
\begin{aligned}
& {\left[\begin{array}{cccccc}
1 & 1 & 1 & 1 & 11 & \\
\mathrm{e}^{-m_{1} L} & \mathrm{e}^{-m_{2} L} & \mathrm{e}^{-m_{3} L} & \mathrm{e}^{m_{1} L} & \mathrm{e}^{m_{2} L} & \mathrm{e}^{m_{3} L} \\
m_{1}^{2} & m_{2}^{2} & m_{3}^{2} & m_{1}^{2} & m_{2}^{2} & m_{3}^{2} \\
m_{1}^{2} \mathrm{e}^{-m_{1} L} & m_{2}^{2} \mathrm{e}^{-m_{2} L} & m_{3}^{2} \mathrm{e}^{-m_{3} L} & k_{1} \mathrm{e}^{m_{1} L} & m_{2}^{2} \mathrm{e}^{m_{2} L} & m_{3}^{2} \mathrm{e}^{m_{3} L} \\
H_{1} & H_{2} & H_{3} & H_{1} & H_{2} & H_{3} \\
-m_{1} H_{1} \mathrm{e}^{-m_{1} L} & -m_{2} H_{2} \mathrm{e}^{-m_{2} L} & -m_{3} H_{3} \mathrm{e}^{-m_{3} L} & m_{1} H_{1} \mathrm{e}^{m_{1} L} & m_{2} H_{2} \mathrm{e}^{m_{2} L} & m_{3} H_{3} \mathrm{e}^{m_{3} L}
\end{array}\right]} \\
& \left\{\begin{array}{l}
C_{1} \\
C_{2} \\
C_{3} \\
C_{4} \\
C_{5} \\
C_{6}
\end{array}\right\}=\left\{\begin{array}{c}
0 \\
0 \\
0 \\
0 \\
\bar{G}(s)+H_{4} \\
0
\end{array}\right\}
\end{aligned}
$$

It is difficult to get an inversion to the Laplace transform of the complicated solutions for the lateral vibration, displacement, thermodynamic temperature, stress and strain in the Laplace transform space. Therefore, the results will be analyzed numerically using a method based on the Fourier series expansion technique. 


\section{Numerical results}

This Section is devoted to investigate some numerical examples of field quantities using material properties of silicon as

$$
\begin{array}{lrr}
E=169 \mathrm{GPa} & \rho=2330 \mathrm{~kg} / \mathrm{m}^{3} & C_{E}=713 \mathrm{~J} /(\mathrm{kg} \mathrm{K}) \\
\alpha_{T}=2.59 \cdot 10^{-9}(1 / \mathrm{K}) & \nu=0.22 & K=156 \mathrm{~W} /(\mathrm{m} \mathrm{K})
\end{array}
$$

The environment temperature of the nanobeam is considered as $T_{0}=293 \mathrm{~K}$. In addition, some parameters like the length-to-thickness $(L / h)$ ratio, width-to-thickness $(b / h)$ ratio, magnitude $\left(q_{0}\right)$, frequency $(\Omega)$ of the applied load and the dimensionless nonlocal parameter $\bar{\xi}$ are fixed to be

$$
\frac{L}{h}=10 \quad \frac{b}{h}=\frac{1}{2} \quad q_{0}=1 \cdot 10^{-8} \quad \Omega=0.1076 \quad \bar{\xi}=10^{6} \xi
$$

Also, Figures 2-5 are plotted by considering the non-dimensional forms appearing in Eqs. (3.3) and (3.4) for a wide range of nanobeam length taken into account that

$$
L=1 \quad t=0.1 \quad z=\frac{h}{3}
$$

(a)

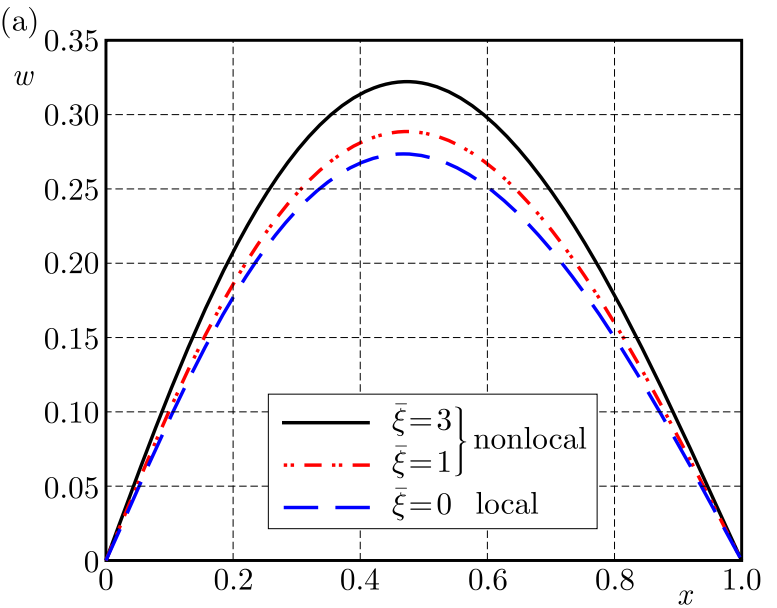

(c)

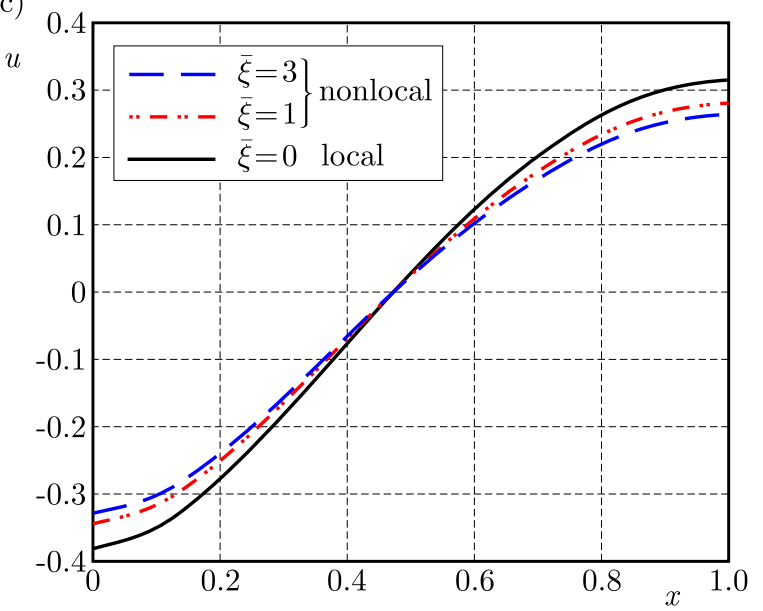

(b)

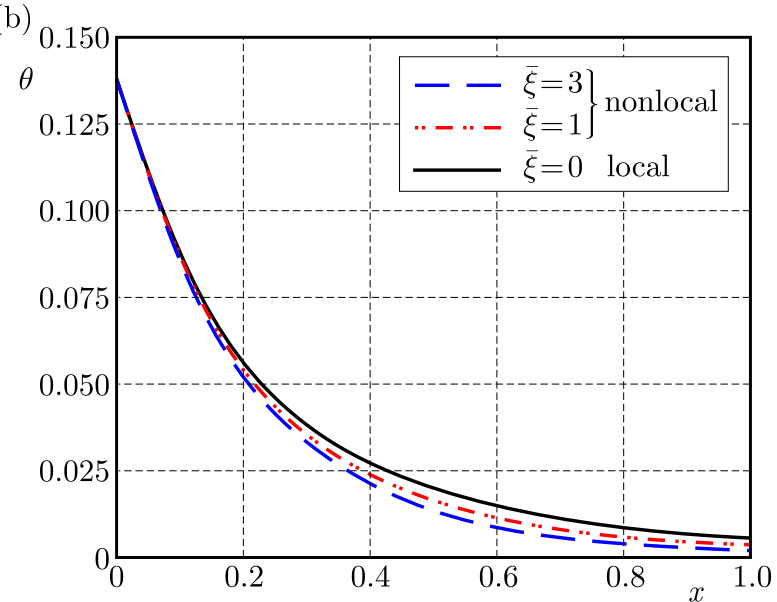

(d)

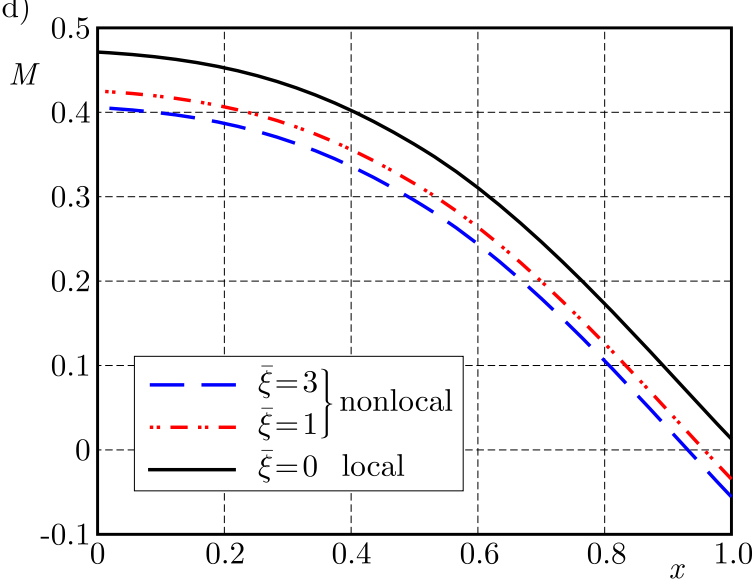

Fig. 2. The transverse deflection, temperature, displacement and bending moment distributions of the nanobeam for different values of the nonlocal thermoelastic parameters $\bar{\xi}$ : (a) transverse

deflection $w$ versus $x$, (b) temperature $\theta$ versus $x$, (c) displacement $u$ versus $x$,

(d) bending moment $M$ versus $x$ 
(a)

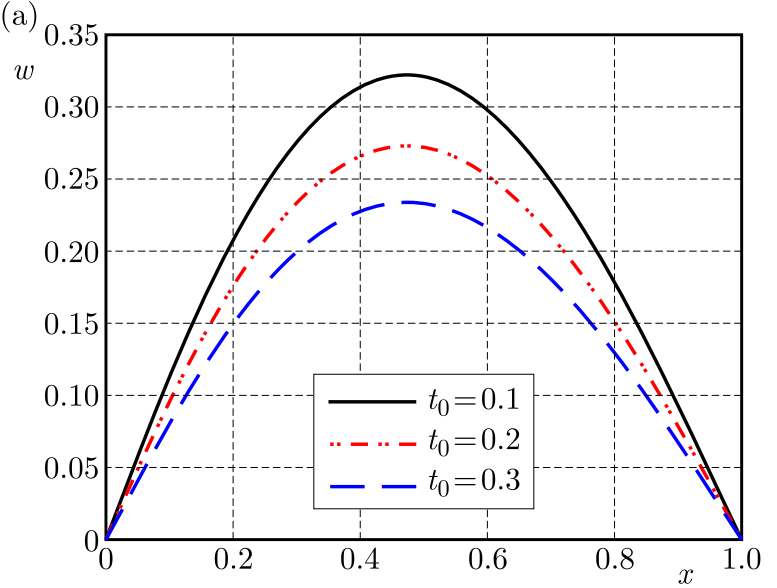

(c)

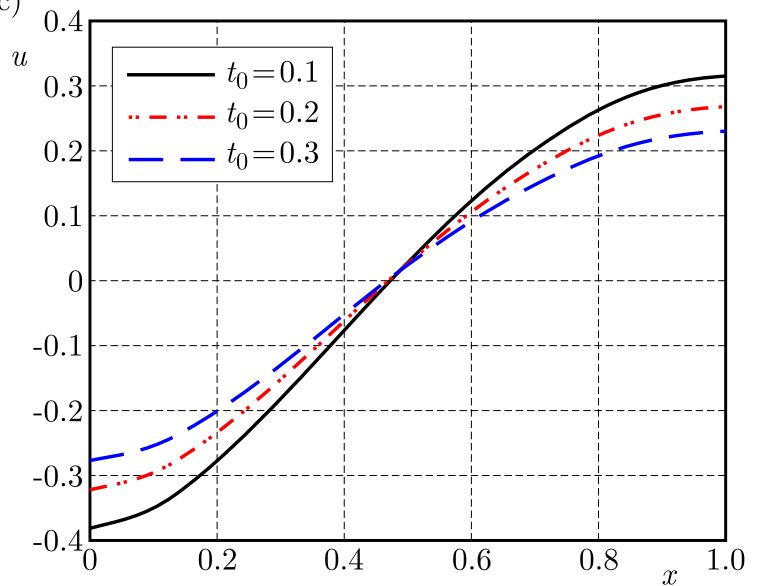

(b)

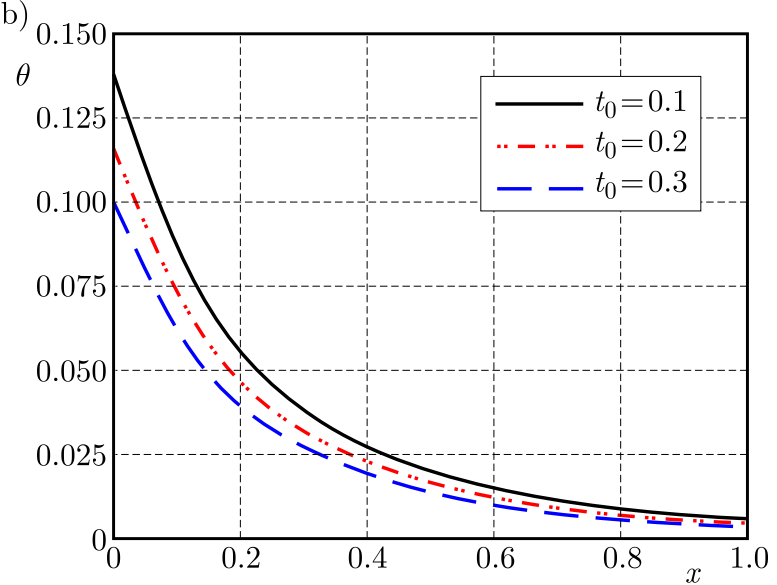

(d)

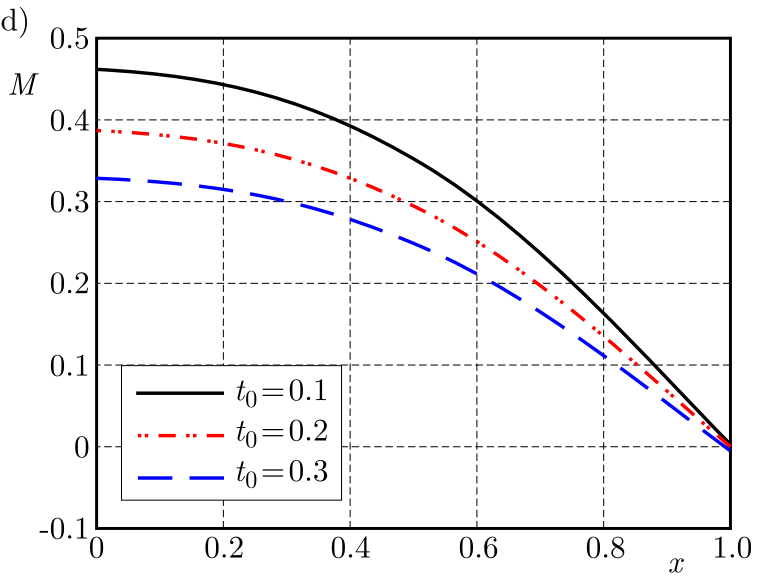

Fig. 3. The transverse deflection, temperature, displacement and bending moment distributions of the nanobeam for different values of the ramping time parameter $t_{0}$ : (a) transverse deflection $w$ versus $x$,

(b) temperature $\theta$ versus $x$, (c) displacement $u$ versus $x$, (d) bending moment $M$ versus $x$

The first example discussed here is to investigate the dimensionless forms of lateral vibration (deflection), temperature, axial displacement and bending moment with different dimensionless nonlocal parameters $\bar{\xi}$. In this example, one considers the ramping time parameter $t_{0}=0.1$ and phase-lags $\tau_{q}$ and $\tau_{\theta}$ remaining constants $\left(\tau_{q}=0.02, \tau_{\theta}=0.01\right)$. It is clear that the case of $\bar{\xi}=0$ indicates the local thermoelasticity theory. However, the values $\bar{\xi}=1$ and $\bar{\xi}=3$ indicate the nonlocal thermoelasticity theory. The effect of $\bar{\xi}$ on vibration characteristics along the axial direction of the nanobeam is shown in Figs. 2a-2d. From these figures, we can see that:

1) The deflection $w$ is no longer increasing and vanishes again at the boundaries. Its maximum values occur near the center of the nanobeam.

2) The lateral vibration $w$ decreases as the nonlocal parameter $\bar{\xi}$ increases.

3) The nanobeam exhibits the maximum deflection near the mid-point of the nanobeam at all times and irrespective of the value of $\bar{\xi}$.

4) The thermal temperature $\theta$ is decreasing as the distance $x$ increases and it is moving in the direction of wave propagation.

5) As $\bar{\xi}$ increases, the temperature $\theta$ decreases.

6) Distribution of the axial displacement $u$ starts increasing with the nonlocal parameter $\bar{\xi}$ in the interval $0 \leqslant x \leqslant 0.45$, thereafter it increases up to maximum amplitudes in the interval $0.45 \leqslant x \leqslant 1$. 
7) The bending moment $M$ is decreasing with the increasing distance $x$. Also, we observe that an increase in $\bar{\xi}$ leads to growth of the distribution of $M$.

8) All plots show that this parameter has a significant effect on the field quantities.

9) It is obvious that according to the value of $\bar{\xi}$, the difference between the local and nonlocal generalized thermoelasticity theory is evident.

The second example discussed here is to investigate the variation of dimensionless lateral vibration, temperature, displacement and bending moment versus the ramping time parameter $t_{0}$ when the phase-lags $\tau_{q}$ and $\tau_{\theta}$ and nonlocal parameter $\bar{\xi}$ remain fixed. The plots of this example are illustrated in Figs. 3a-3d. It is concluded in this case that:

1) Values of the deflection, temperature and bending moment are increasing as the ramping time parameter $t_{0}$ is decreasing.

2) As $t_{0}$ increases, the displacement $u$ increases in the interval $0 \leqslant x<0.48$ and decreases in the interval $0.48<x \leqslant 1$. The axial displacement $u$ increases along the axial direction.

3) The distribution of all variables is very sensitive to the variation of the ramping time parameter $t_{0}$.
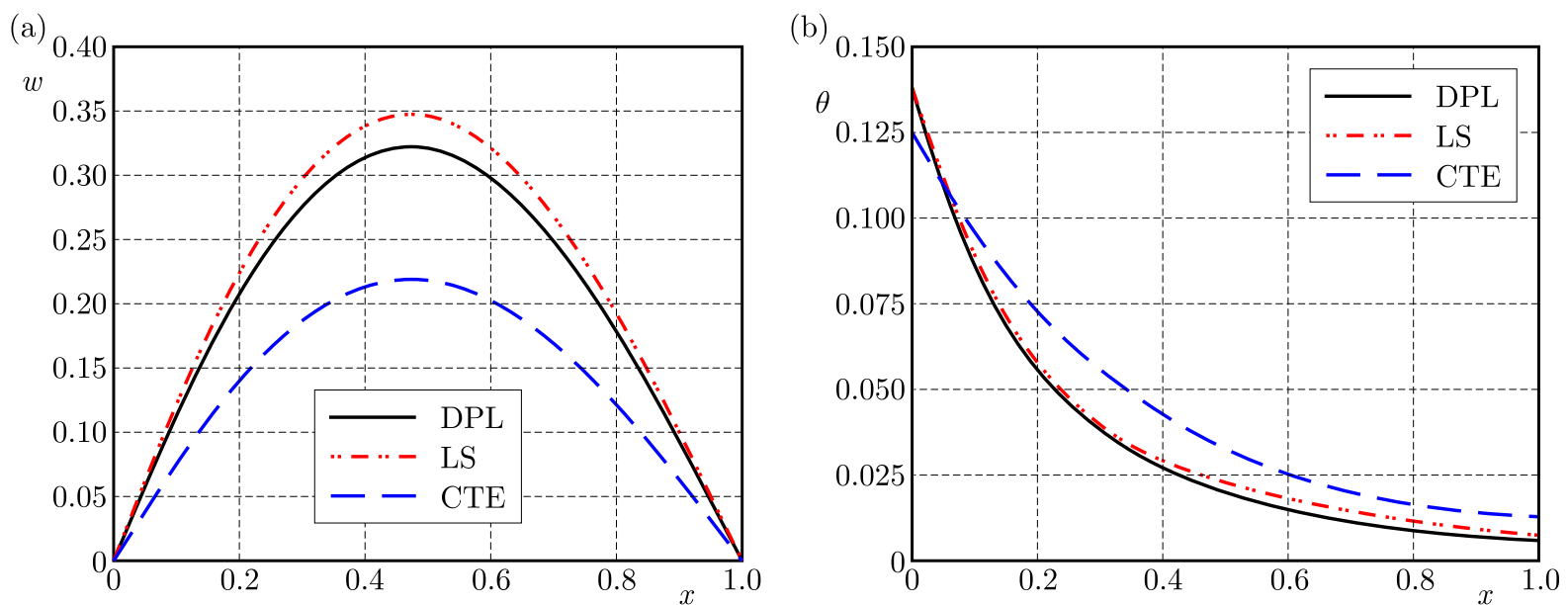

(c)

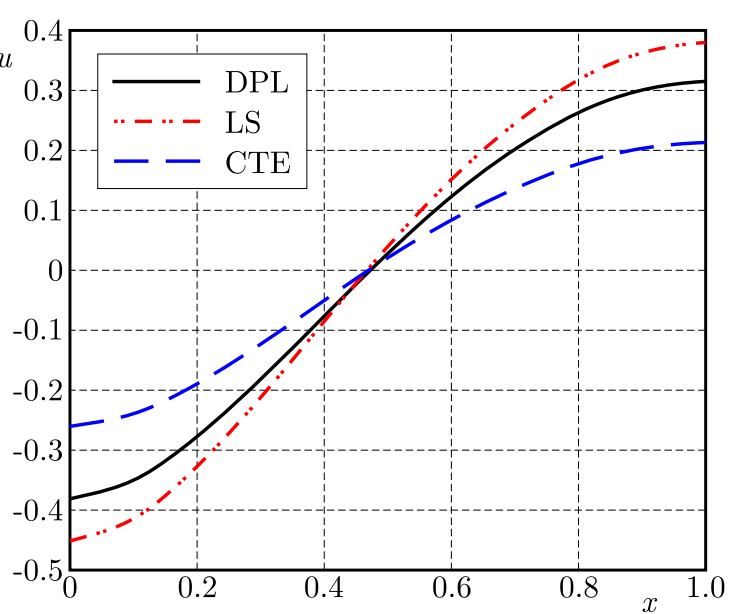

(d)

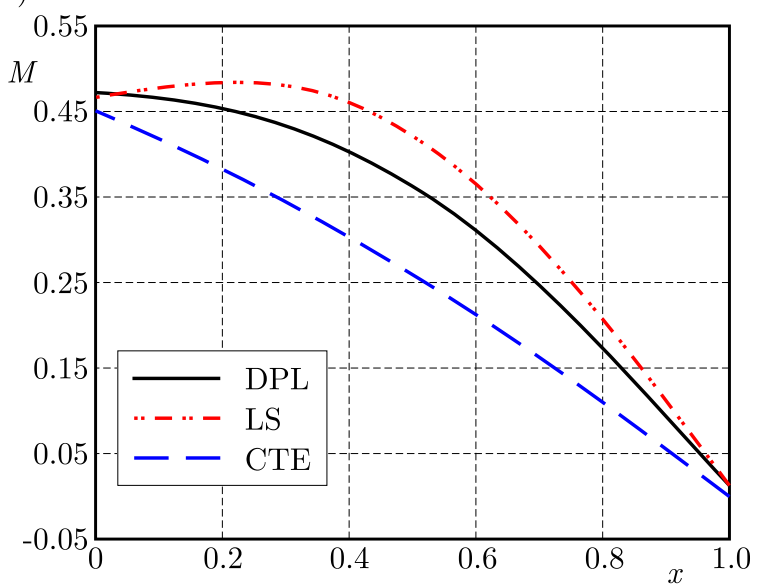

Fig. 4. The transverse deflection, temperature, displacement and bending moment distributions of the nanobeam for different values of the phase lags $\tau_{q}$ and $\tau_{\theta}$ : (a) transverse deflection $w$ versus $x$,

(b) temperature $\theta$ versus $x$, (c) displacement $u$ versus $x$, (d) bending moment $M$ versus $x$

In the third example, Figs. 4a-4d are plotted to give a comparison of the results obtained for dimensionless lateral vibration, temperature, displacement and bending moment distributions 
for different values of $\tau_{q}$ and $\tau_{\theta}$ at $t_{0}=0.2$ and $\bar{\xi}=2$. The graphs in Figs. 4a-4d represent four curves predicted by two thermoelasticity theories, the coupled theory (CTE) and the Lord-Shulman theory (LS), obtained as special cases of the dual-phase-lag model. The computations have been performed for one value of time, namely for $t=0.12$ and various values of the parameters $\tau_{q}$ and $\tau_{\theta}$. The coupled theory (CTE) is given by setting $\left(\tau_{\theta}=\tau_{q}=0\right)$, the LordShulman theory (LS) is given by putting $\left(\tau_{\theta}=0\right.$ and $\left.\tau_{q}=\tau_{0}>0\right)$ and the DPL is given by setting $\tau_{q}>0$ and $\tau_{\theta}>0$. It can be found from Figs. 4a-4d that the distribution in LS model is near to that in DPL theory, whereas the distributions in CTE theory are different from that in DPL theory. Also, values of $\tau_{q}$ and $\tau_{\theta}$ can judge whether the wavelike behavior in the phase-lag heat conduction is dominant or not.

(a)

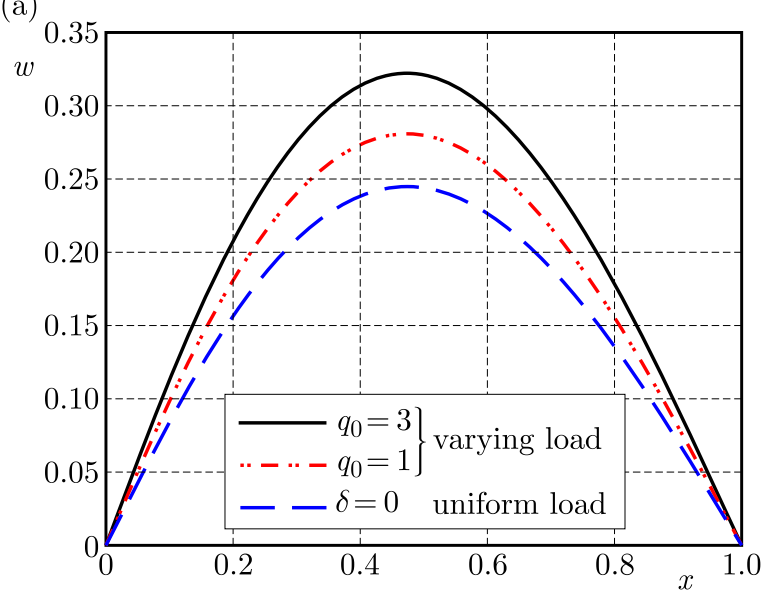

(c)

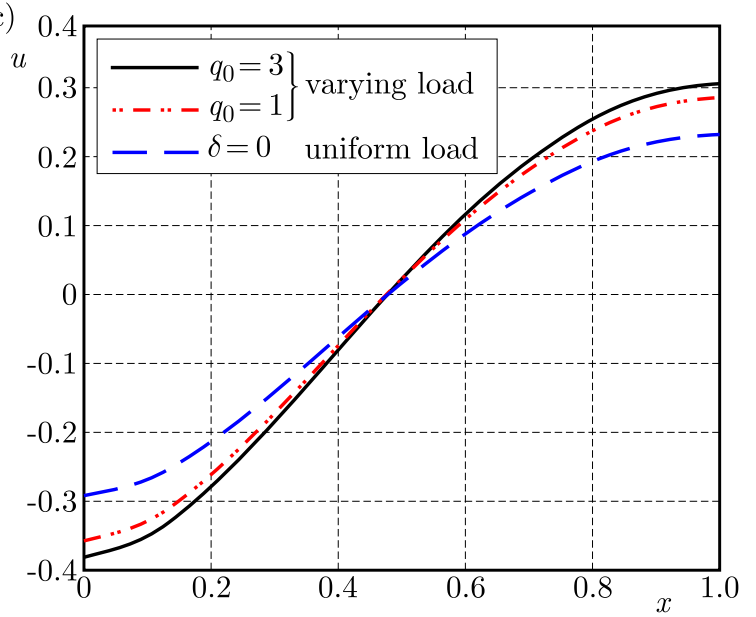

(b)

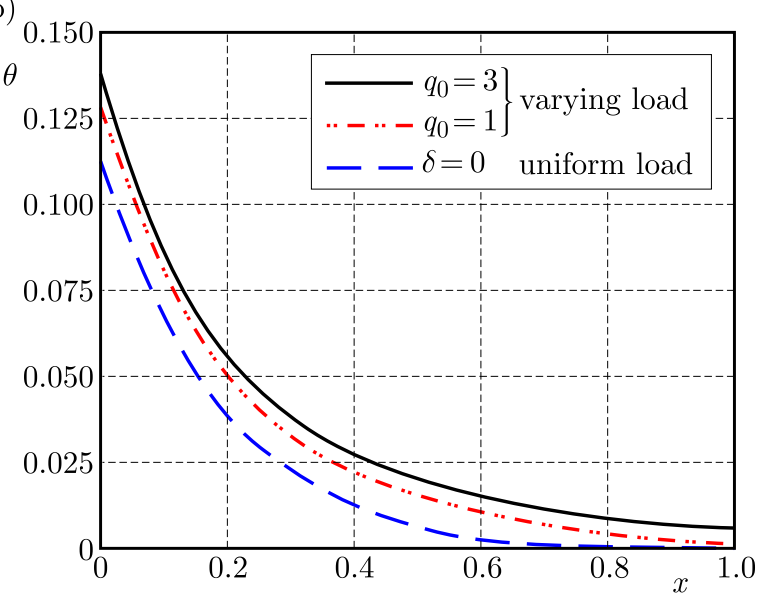

(d)

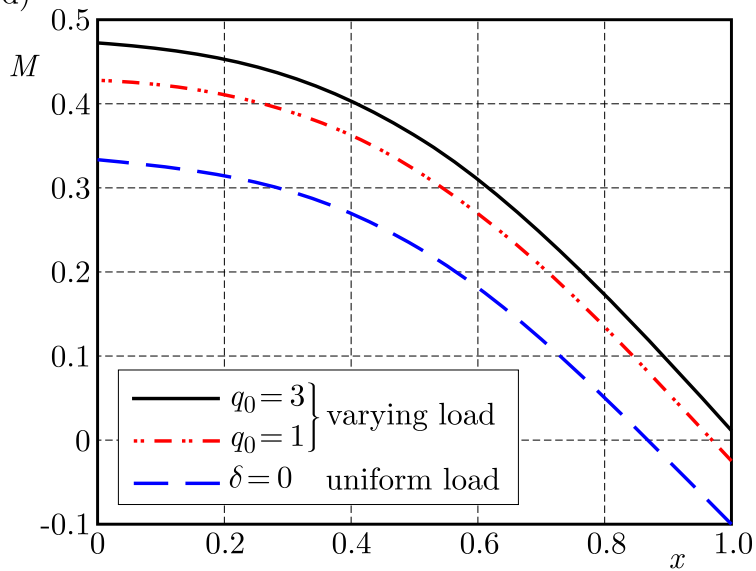

Fig. 5. The transverse deflection, temperature, displacement and bending moment distributions of the nanobeam for different values of the point load $q_{0}$ : (a) transverse deflection $w$ versus $x$,

(b) temperature $\theta$ versus $x$, (c) displacement $u$ versus $x$, (d) bending moment $M$ versus $x$

In the last example, three different values of the dimensionless magnitude of the point load $q_{0}$ are considered. For a uniformly distributed load, we put $\delta=0$, and for an exponential decaying time varying load, we take $\delta=1$. We found from Figs. 5a-5d that, as the point load $q_{0}$ increases, the values of lateral vibration, temperature, moment fields are also increasing. Once again, the displacement $u$ increases in the interval $0 \leqslant x<0.48$ and decreases in the interval $0.48<x \leqslant 1$. Finally, all variables are very sensitive to the variation of the point load $q_{0}$. 


\section{Conclusions}

In this work, a mathematical model that governs the nonlocal generalized theory of thermoelasticity with phase lags for nanobeams subjected to dynamical transverse loads is established. The first boundary of the nanobeam is subjected to a ramp-type heating. The Laplace transformation numerical technique has been used. The effects of dynamic loads $q_{0}$, nonlocal parameter $\bar{\xi}$ and ramping time parameter $t_{0}$ on all quantities are investigated and illustrated graphically. One can conclude, according to the numerical results shown in all figures, that:

- The nonlocal $\bar{\xi}$ and ramping time $t_{0}$ parameters have significant effects on all quantities.

- Some quantities like thermoelastic moment, displacement and temperature are strongly dependent on the ramping time parameter $t_{0}$.

- A phenomenon of finite speeds of propagation is observed in all depicted figures. This is predictable since the thermal wave travels with a finite speed.

- The effects of dynamic loads on all the studied fields are very significant.

- Significant differences in the physical quantities are observed between the exponential decaying time varying load and the uniformly distributed load.

- Vibration of nanotubes is an important subject in the study of nanotechnology since it relates to electronic and optical properties of multiwall carbon nanotubes.

- This study is required for researchers and designers to be applied in the design and development of different devices, especially those being under environmental loads, likes resonators.

\section{References}

1. Abbas I.A., Zenkour A.M., 2014, Dual-phase-lag model on thermoelastic interactions in a semi-infinite medium subjected to a ramp-type heating, Journal of Computational and Theoretical Nanoscience, 11, 3, 642-645

2. Abouelregal A.E., 2011, Generalized thermoelasticity for an isotropic solid sphere in dual-phase-lag of heat transfer with surface heat flux, International Journal for Computational Methods in Engineering Science and Mechanics, 12, 96-105

3. Abouelregal A.E., Abo-Dahab S.M., 2012, Dual phase lag model on magneto-thermoelasticity infinite non-homogeneous solid having a spherical cavity, Journal of Thermal Stresses, 35, 820-841

4. Allamen S.M., 2003, An introduction to mechanical-properties-related issues in MEMS structures, Journal of Materials Scienc, 38, 4115-4123

5. Arefi M., Zenkour A.M., 2016, A simplified shear and normal deformations nonlocal theory for bending of functionally graded piezomagnetic sandwich nanobeams in magneto-thermo-electric environment, Journal of Sandwich Structures and Materials, 18, 5, 624-651

6. Eringen A.C., 1983, On differential equations of nonlocal elasticity and solutions of screw dislocation and surface waves, Journal of Applied Physics, 54, 4703-4710

7. Eringen A.C., 2002, Nonlocal Continuum Field Theories, New York: Springer Verlag

8. Lord H.W., Shulman Y., A generalized dynamical theory of thermoelasticity, Journal of the Mechanics and Physics of Solids, 15, 299-309

9. Tzou D.Y., 1995, A unified approach for heat conduction from macro- to micro-scales, Journal of Heat Transfer, 117, 8-16

10. Tzou D.Y., 1997, Macro-to-Micro Scale Heat Transfer: the Lagging Behavior, Taylor and Francis, Washington (DC) 
11. Younis M.I., 2011, MEMS Linear and Non-linear Statics and Dynamics, Springer, New York, USA, 2011

12. Zenkour A.M., 2016a, Nonlocal transient thermal analysis of a single-layered graphene sheet embedded in viscoelastic medium, Physica E, 79, 87-97

13. Zenkour A.M., 2016b, Two-dimensional coupled solution for thermoelastic beams via generalized dual-phase-lags model, Mathematical Modelling and Analysis, 21, 3, 319-335

14. Zenkour A.M., 2016c, Vibration analysis of a single-layered graphene sheet embedded in viscoPasternak's medium using nonlocal elasticity theory, Journal of Vibroengineering, 18, 4, 2319-2330

15. Zenkour A.M., Abouelregal A.E., 2014a, Nonlocal thermoelastic vibrations for variable thermal conductivity nanobeams due to harmonically varying heat, Journal of Vibroengineering, 16, $8,3665-3678$

16. Zenkour A.M., Abouelregal A.E., 2014b, Vibration of FG nanobeams induced by sinusoidal pulse-heating via a nonlocal thermoelastic model, Acta Mechanica, 225, 12, 3409-3421

17. Zenkour A.M., Abouelregal A.E., 2015, Nonlocal thermoelastic nanobeam subjected to a sinusoidal pulse heating and temperature-dependent physical properties, Microsystem Technologies, 21, 8, 1767-1776

18. Zenkour A.M. And Abouelregal A.E., 2016, Nonlinear effects of thermo-sensitive nanobeams via a nonlocal thermoelasticity model with relaxation time, Microsystem Technologies, 22, 10, $2407-2415$

19. Zenkour A.M., Mashat D.S., Abouelregal A.E., 2013, The effect of dual-phase-lag model on reflection of thermoelastic waves in a solid half space with variable material properties, Acta Mechanica Solida Sinica, 26, 6, 659-670

20. Zhang Y.Q., LiU G.R., Xie X.Y., 2005, Free transverse vibrations of double-walled carbon nanotubes using a theory of nonlocal elasticity, Physical Review B, 71, 195 404, 2005. 